KAIST-CHEP-94-07

YUMS-94-05

\title{
Polyakov's Spin Factor for a Classical Spinning Particle via BRST Invariant Path Integral
}

\author{
Jin-Ho Cho 网 \\ Department of Physics \\ Korea Advanced Institute of Science and Technology \\ 373-1 Yusung-ku, Taejon, 305-701, Korea \\ Seungjoon Hyun ind Hyuk-jae Lee \\ Institute for Mathematical Sciences \\ Yonsei University, Seoul, 120-749, Korea
}

\begin{abstract}
For the 'classical' formulation of a massive spinning particle, the propagator is obtained along with the spin factor. We treat the system with two kinds of constraints that were recently shown to be concerned with the reparametrization invariance and 'quasi-supersymmetry'. In the path integral, the BRST invariant Lagrangian is used and the same spin factor is obtained as in the pseudo-classical formulation.
\end{abstract}

*jhcho@chiak.kaist.ac.kr

†hyun@phya.yonsei.ac.kr

‡lhjae@bubble.yonsei.ac.kr 
There are two standard ways of describing the spin degrees of freedom for the relativistic particle; the 'classical' way describes them in terms of the Lorentz group elements [1] and the 'pseudo-classical' one does that in terms of the Grassmann quantities [2]. The main difference between them is the symmetries. The classical system for the relativistic particle has the reparametrization symmetry while the pseudo-classical one has extra supersymmetry.

In Ref. [3], it is shown that this difference is due to the constraint structure of the systems; the constraint $p_{\mu} \cdot S^{\mu \nu}=0$ for the classical system [4 can be relaxed without changing the physical properties of the system. Furthermore that constraint, which can be considered as the supersymmetry analogue of the classical system, gives a closed algebra together with the constraint $p^{2}+m^{2}=0$.

In this letter, we are to confirm again that the model gives the right description of the relativistic spinning particle through the BRST invariant construction of the propagator along with the spin factor.

The model is given by the following Lagrangian

$$
\begin{aligned}
\mathcal{L}= & p^{\mu} \dot{x}_{\nu}-\frac{\lambda}{2}\left(t_{1} \dot{t}_{2}-t_{2} \dot{t}_{1}\right)-\frac{\lambda}{2}\left(\Lambda_{\mu 1} \dot{\Lambda}^{\mu}{ }_{2}-\Lambda_{\mu 2} \dot{\Lambda}^{\mu}{ }_{1}\right) \\
& -M_{1}\left(\frac{p_{\mu}}{m} \Lambda_{2}^{\mu}+t_{2}\right)+M_{2}\left(\frac{p_{\mu}}{m} \Lambda^{\mu}{ }_{1}+t_{1}\right)-N\left(p^{2}+m^{2}\right) \\
\equiv & p^{\mu} \dot{x}_{\mu}-\lambda t_{1} \dot{t}_{2}-\lambda u \cdot \dot{v}-M_{1} \Phi_{1}-M_{2} \Phi_{2}-N \Phi_{N}
\end{aligned}
$$

where $u^{2}=1, v^{2}=1$ and $u \cdot v=0$ ?. From this first order Lagrangian the Poisson brackets are easily shown to be

$$
\begin{aligned}
& \left\{x^{\mu}, p^{\nu}\right\}=\eta^{\mu \nu}, \quad\left\{t_{1}, t_{2}\right\}=\frac{1}{\lambda}, \\
& \left\{u^{\mu}, v^{\nu}\right\}=\frac{1}{\lambda} \eta^{\mu \nu},
\end{aligned}
$$

\footnotetext{
${ }^{1}$ we set $\lambda^{\mu \nu}$ of $[3]$ equal to $\lambda \delta_{1}^{\mu} \delta_{2}^{\nu}$ for our convenience.
} 
and the constraint algebra is given by

$$
\begin{aligned}
\left\{\Phi_{N}, \Phi_{N}\right\} & =\left\{\Phi_{N}, \Phi_{i}\right\}=0 \\
\left\{\Phi_{i}, \Phi_{j}\right\} & =-\frac{\epsilon_{i j}}{\lambda m^{2}} \Phi_{N}, \quad i, j=1,2
\end{aligned}
$$

They form a closed algebra similar to the supersymmetry of the pseudo-classical formulation. Here, we do not treat the group relation $u^{2}=1, v^{2}=1$ and $u \cdot v=0$ as the 'dynamical' constraints 2. Being absorbed into the measure of the path integral they do the role of reducing the integration range.

The Lagrangian can be written in the second order style

$$
\mathcal{L}=-m \sqrt{-\left(\dot{x}-\frac{\lambda}{m}\left(v \dot{t}_{1}-u \dot{t}_{2}\right)\right)^{2}}+\lambda t_{1} \dot{t}_{2}-\lambda u \cdot \dot{v},
$$

in which $N^{2}=-\frac{1}{4 m^{2}}\left(\dot{x}-\frac{\lambda}{m}\left(v \dot{t}_{1}-u \dot{t}_{2}\right)\right)^{2}$ (this comes from the equation of motion for $p$ ) is used. We choose $N$ to be positive for later use.

In order to deal with the gauge invariant system given above, we follow the standard BRST formulation [5]. For the constraints above (3), we straightforwardly construct the BRST operator [6]

$$
Q=c_{N} \Phi_{N}+c_{i} \Phi_{i}+\frac{1}{\lambda m^{2}} c_{1} c_{2} \chi_{N}+\pi_{N} \bar{\chi}_{N}+\pi_{1} \bar{\chi}_{1}+\pi_{2} \bar{\chi}_{2}, \quad i=1,2
$$

and the gauge fermion

$$
\psi=\chi_{N} N+\chi_{1} M_{1}+\chi_{2} M_{2}
$$

which is corresponding to the usual gauge fixing $\dot{N}=\dot{M}_{1}=\dot{M}_{2}=0$. Here $\left(c_{a}, \bar{\chi}_{a}\right)$ and $\left(\chi_{a}, \bar{c}_{a}\right)$ are ghosts and anti-ghosts, respectively. We use the following Poisson brackets for ghost variables:

$$
\begin{aligned}
& \left\{N, \pi_{N}\right\}=\left\{M_{i}, \pi_{i}\right\}=1, \quad i=1,2 \\
& \left\{c_{a}, \chi_{a}\right\}=1, \quad\left\{\bar{c}_{a}, \bar{\chi}_{a}\right\}=1, \quad a=1,2, N .
\end{aligned}
$$

\footnotetext{
${ }^{2}$ in ref. [3, they are thus dealt with and result in similar quasi-supersymmetry although in a somewhat complicated form.
} 
The BRST invariant Hamiltonian is

$$
\begin{aligned}
H_{Q}= & \{\psi, Q\} \\
= & N \Phi_{N}+M_{1} \Phi_{1}+M_{2} \Phi_{2}+\frac{1}{\lambda m^{2}} c_{2} M_{1} \chi_{N} \\
& -\frac{1}{\lambda m^{2}} c_{1} M_{2} \chi_{N}+\chi_{N} \bar{\chi}_{N}+\chi_{1} \bar{\chi}_{1}+\chi_{2} \bar{\chi}_{2} .
\end{aligned}
$$

The effective BRST invariant Lagrangian is obtained through the Legendre transformation as follows

$$
\begin{aligned}
\mathcal{L}_{e f f}= & p \cdot \dot{x}-\lambda t_{1} \dot{t}_{2}-\lambda u \dot{v}+\dot{N} \pi_{N}+\dot{M}_{1} \pi_{1}+\dot{M}_{2} \pi_{2} \\
& +\dot{c}_{N} \chi_{N}+\dot{c}_{1} \chi_{1}+\dot{c}_{2} \chi_{2}-\dot{\bar{c}}_{N} \bar{\chi}_{N}-\dot{\bar{c}}_{1} \bar{\chi}_{1}-\dot{\bar{c}}_{2} \bar{\chi}_{2}-H_{Q}
\end{aligned}
$$

where the canonical kinetic terms for the ghost variables are assumed. For later convenience we divide the whole Lagrangian into two parts; the matter part

$$
\begin{aligned}
\mathcal{L}_{\text {matter }}= & p \cdot \dot{x}-\lambda t_{1} \dot{t}_{2}-\lambda u \dot{v}+\dot{N} \pi_{N}+\dot{M}_{1} \pi_{1}+\dot{M}_{2} \pi_{2} \\
& -N\left(p^{2}+m^{2}\right)-M_{1}\left(\frac{p}{m} \cdot v+t_{2}\right)-M_{2}\left(\frac{p}{m} \cdot u+t_{1}\right),
\end{aligned}
$$

and the ghost part

$$
\begin{aligned}
\mathcal{L}_{\text {ghost }}= & \dot{c}_{N} \chi_{N}+\dot{c}_{1} \chi_{1}+\dot{c}_{2} \chi_{2}-\dot{\bar{c}}_{N} \bar{\chi}_{N}-\dot{\bar{c}}_{1} \bar{\chi}_{1}-\dot{\bar{c}}_{2} \bar{\chi}_{2} \\
& -\frac{1}{\lambda m^{2}} c_{2} M_{1} \chi_{N}+\frac{1}{\lambda m^{2}} c_{1} M_{2} \chi_{N}-\chi_{N} \bar{\chi}_{N}-\chi_{1} \bar{\chi}_{1}-\chi_{2} \bar{\chi}_{2} .
\end{aligned}
$$

Now we are to consider transition amplitude via Feynman path integral. The matter part is written as

$$
\begin{aligned}
Z_{\text {matter }}= & \int D p D x D t_{1} D t_{2} \tilde{D} u \tilde{D} v D N D M_{1} D M_{2} D \pi_{N} D \pi_{1} D \pi_{2} \delta[u \cdot v] \\
& \cdot \exp \left\{-i \int d \tau\left[p \cdot \dot{x}-\lambda t_{1} \dot{t}_{2}-\lambda u \dot{v}+\dot{N} \pi_{N}+\dot{M}_{1} \pi_{1}+\dot{M}_{2} \pi_{2}\right.\right. \\
& \left.\left.-N\left(p^{2}+m^{2}\right)-M_{1}\left(\frac{p}{m} \cdot v+t_{2}\right)-M_{2}\left(\frac{p}{m} \cdot u+t_{1}\right)\right]\right\},
\end{aligned}
$$

where $\tilde{D} u \tilde{D} v=D u D v \delta\left[u^{2}-1\right] \delta\left[v^{2}-1\right]$. The integration over $\pi_{a}, a=1,2, N$ gives the delta functionals $\delta[\dot{N}], \delta\left[\dot{M}_{1}\right]$ and $\delta\left[\dot{M}_{2}\right]$, which result in converting those functional 
measures $D N D M_{1} D M_{2}$ into the ordinary ones $d N d M_{1} d M_{2}$. The same is true for the integration over $x$ and the measure $D p$ turns into $d p$. The ordinary integration over $N$, which is considered to be positive, can be performed straightforwardly to give the usual propagator for the relativistic particle with appropriate regularization.

$$
\int d N e^{-i \int d \tau N\left(p^{2}+m^{2}\right)}=\int d N \exp \left\{-i T N\left(p^{2}+m^{2}\right)\right\}=\frac{-i}{\left(p^{2}+m^{2}-i \varepsilon\right) T}
$$

where $T=\tau_{f}-\tau_{i}$. Now the matter part may be rewritten as

$$
\begin{aligned}
Z_{\text {matter }}= & \int d p d M_{1} d M_{2} D t_{1} D t_{2} \tilde{D} u \tilde{D} v \delta[u \cdot v] \frac{i}{\left(p^{2}+m^{2}-i \varepsilon\right) T} \\
& \cdot \exp \left\{-i \int d \tau\left[\lambda t_{1} \dot{t}_{2}+\lambda u \cdot \dot{v}+M_{1} \Phi_{1}+M_{2} \Phi_{2}\right]\right\}
\end{aligned}
$$

The ghost part reads as

$$
\begin{aligned}
Z_{\text {ghost }}= & \int D c D \chi D \bar{c} D \bar{\chi} \\
& \cdot \exp \left\{i \int d \tau\left[\dot{c}_{a} \chi_{a}-\dot{\bar{c}}_{a} \bar{\chi}_{a}-\chi_{a} \bar{\chi}_{a}-\frac{1}{\lambda m^{2}} c_{2} M_{1} \chi_{N}+\frac{1}{\lambda m^{2}} c_{1} M_{2} \chi_{N}\right]\right\} \\
= & \int D c D \chi D \bar{c} D \bar{\chi} \\
& \cdot \exp \left\{i \int d \tau\left[-\left(\chi_{a}+\dot{\bar{c}}_{a}\right)\left(\bar{\chi}_{a}+\dot{c}_{a}-f_{b c}^{a} c_{b} M_{c}\right)+\dot{\bar{c}}_{a}\left(\dot{c}_{a}-f_{b c}^{a} c_{b} M_{c}\right)\right]\right\}
\end{aligned}
$$

where the structure constant $f_{b c}^{a}$ is nonvanishing only for the following component

$$
f_{i j}^{N}=-\frac{\epsilon_{i j}}{\lambda m^{2}}
$$

This Gaussian integration can be performed easily by the field shifting

$$
\begin{aligned}
Z_{\text {ghost }} & =\int D c D \chi D \bar{c} D \bar{\chi} \exp \left\{i \int d \tau\left(-\chi_{a} \bar{\chi}_{a}+\dot{\bar{c}}_{a} \dot{c}_{a}\right)\right\} \\
& =\operatorname{const}(i T)^{3} .
\end{aligned}
$$

We note that this ghost part has only $T$ dependency. 
Now we are in a position to finish the remaining integration for the matter part.

$$
\begin{aligned}
Z_{\text {matter }}= & \int d p d M_{1} d M_{2} D t_{1} D t_{2} \tilde{D} u \tilde{D} v \delta[u \cdot v] \frac{-i}{\left(p^{2}+m^{2}-i \varepsilon\right) T} \\
& \cdot \exp \left\{-i \int d \tau\left[\lambda t_{1} \dot{t}_{2}+\lambda u \cdot \dot{v}+M_{1}\left(\frac{p}{m} \cdot v+t_{2}\right)+M_{2}\left(\frac{p}{m} \cdot u+t_{1}\right)\right]\right\} .
\end{aligned}
$$

The integration over $t_{1}$ gives delta functional

$$
\int D t_{1} e^{-i \int d \tau t_{1}\left(\lambda \dot{t}_{2}+M_{2}\right)}=\delta\left[\lambda \dot{t}_{2}+M_{2}\right]
$$

which converts the functional integral over $t_{2}$ into the ordinary one over the constant mode $t_{2 i}$ given as

$$
t_{2}(\tau)=-\frac{M_{2}}{\lambda} \tau+\frac{M_{2}}{\lambda} \tau_{i}+t_{2 i}
$$

With the substitution of (20), the matter part is summarized as

$$
\begin{aligned}
Z_{\text {matter }}= & \int d p d M_{1} d M_{2} d t_{2 i} \tilde{D} u \tilde{D} v \delta[u \cdot v] \frac{-i}{\left(p^{2}+m^{2}-i \varepsilon\right) T} \\
& \cdot e^{-i \int d \tau\left[\lambda u \cdot \dot{v}+M_{1}\left(-\frac{M_{2}}{\lambda} \tau+\frac{M_{2}}{\lambda} \tau_{i}+t_{2 i}\right)-M_{1} \frac{p}{m} \cdot v+M_{2} \frac{p}{m} \cdot u\right]} .
\end{aligned}
$$

Finally the integrations over $t_{2 i}$ and $M_{1}$ result in

$$
\int d t_{2 i} \exp \left\{-i M_{1} T t_{2 i}\right\}=\delta\left(M_{1} T\right)
$$

and

$$
\int d M_{1} \delta\left(M_{1} T\right) \exp \left\{-i \int d \tau M_{1}\left(-\frac{M_{2}}{\lambda} \tau+\frac{M_{2}}{\lambda} \tau_{i}+t_{2 i}\right)\right\}=\frac{1}{T} .
$$

Furthermore $\dot{v}$ may be expanded as $\dot{v}=C_{21} u+C_{11} v+w$, where $u \cdot w=v \cdot w=0$, thus the matter part becomes

$$
\begin{aligned}
Z_{\text {matter }}= & \int d p d M_{2} \tilde{D} u \tilde{D} v \delta[u \cdot v] \\
& \cdot \frac{-i}{\left(p^{2}+m^{2}-i \varepsilon\right) T^{2}} \exp \left\{-i \int d \tau\left[\lambda u \cdot \dot{v}+M_{2} \frac{p}{m} \cdot u\right]\right\}
\end{aligned}
$$




$$
\begin{aligned}
= & \int d p d M_{2} \tilde{D} u \tilde{D} v \delta[u \cdot v] \\
& \cdot \frac{-i}{\left(p^{2}+m^{2}-i \varepsilon\right) T^{2}} \exp \left\{-i \int d \tau\left[\lambda C_{21}(\tau)+M_{2} \frac{p}{m} \cdot u\right]\right\} .
\end{aligned}
$$

Since $C_{21}$ has no field dependency it can be extracted out of the functional integral and with the integration over $M_{2}$ (24) becomes

$$
e^{-i \lambda \int d \tau C_{21}} \int d p \tilde{D} u \tilde{D} v \delta[u \cdot v] \frac{-i}{\left(p^{2}+m^{2}-i \varepsilon\right) T^{2}} \delta\left[\int d \tau \frac{p}{m} \cdot u\right] .
$$

Note that the integrand does not have $v$ field dependency thus integral over $v$ part also can be decoupled to be absorbed into the normalization factor. Moreover if $u$ is periodic, it can be mode-expanded thus only the zero mode $u_{0}$ remains in the delta functional $\delta\left[\int d \tau \frac{1}{m} p \cdot u\right]=\delta\left(\frac{T}{m} p \cdot u_{0}\right)$. This implies that along with the integration over $u$ it gives the order $T^{-1}$ which together with $T^{-2}$ in (25) can be exactly cancelled by the $T^{3}$ order of the ghost part (17).

We conclude this letter with some remarks. There have been many studies on the BRST quantization of the relativistic spinning particle. In [7] the massive case is treated, in [8] is dealt with the massless case and in [9] the extended supersymmetric case is considered. However in most cases, they use the pseudo-classical formulation.

In this letter, we have worked the same for the 'classical' system with the relaxed constraint $\frac{1}{m} p_{\mu} \Lambda^{\mu}{ }_{\nu}+t_{\nu}=0$. In the final result (25), we note that the BRST invariance assures the cancellation of the unphysical $T$ dependency of the propagator. Further the spin factor is the same as in [10]. The delta functional $\delta\left[\int d \tau \frac{1}{m} p \cdot u\right]$ says that only those $u$ satisfying $\int d \tau \frac{1}{m} p \cdot u=0$ contribute to the spin factor and becomes some volume factor upon integration. In such a case $\frac{1}{m} p \cdot u$ may not vanish for all time, which is just compatible with the relaxed constraint $\frac{1}{m} p_{\mu} \Lambda^{\mu}{ }_{i}=-t_{i}$. The same is true for $v$ since we can change the role of $u$ and $v$ by the integration by parts in (18). We can say, therefore, the model proposed in [3] describes a spinning particle in the 'classical' fashion comparable with the 'pseudo-classical' formulation. 


\section{References}

[1] A. Hanson, T. Regge and C. Teitelboim, Accademia Nazionale dei Lincei (Roma, 1976).

[2] F.A. Berezin and M.S. Marinov Ann.Phys.104(1977)336; L. Brink, P. Di Vecchia and P. Howe Nucl. Phys. B118(1977)76.

[3] J.-H. Cho, S.J. Hyun and J.-K.Kim, Mod. Phys. Lett. A (To appear).

[4] A.P. Balachandran, G. Marmo, B.-S. Skagerstam and A. Stern, Gauge Symmetries and Fiber Bundles, (Springer-Verlag, Berlin, 1983).

[5] C. Becchi, A. Rouet and R. Stora, Phys. Lett. B52(1974)344; I. V. Tyutin, Lebedev preprint FIAN No.39(1975); T. Kugo and I. Ojima, Suppl. Prog. Theo. Phys.66(1979)1.

[6] E.S. Fradkin and G.A. Vilkovisky, Phys. Lett. B55(1975)224; I.A. Batalin and G.A. Vilkovisky, Phys. Lett. B(1977)309.

[7] A. Barducci, R. Caslbuoni, D. Dominici and R. Gatto, Phys. Lett. B 187 (1987)135; C. Batlle, J. Gomis and J. Roca, Phys. Rev. D40(1989)1950.

[8] A. M. Polyakov, Gauge Fields and Strings,(Harwood, Chur, Switzerland, 1987).

[9] M. Pierri and V. O. Rivelles, Phys. Lett. B251(1990)421; Q. Lin and G. Ni, Phys. Rev. D41(1990)1307.

[10] A. M. Polyakov, Les Houches, Session XLIX,(ed. E. Brézin and J. ZinnJustin,1988); J. Grundberg, T. H. Hansson, A. Karlhede and U. Lindström, Phys. Lett. B218(1989) 321; J. Grundberg, T. H. Hansson, and A. Karlhede, Nucl. Phys. B347(1990)420. 